\title{
Massive cerebral venous sinus thrombosis secondary to Graves' disease
}

\author{
Hye-Min Son \\ Department of Radiology, Keimyung University Dongsan Hospital, Daegu, Korea
}

Received: August 6, 2019

Revised: September 9, 2019

Accepted: September 11, 2019

Corresponding author:

Hye-Min Son

Department of Radiology, Keimyung

University Dongsan Hospital, 1035,

Dalgubeol-daero, Dalseo-gu, Daegu

42601, Korea

Tel: +82-53-250-4120

Fax: +82-53-250-4153

E-mail:shmm11111@gmail.com
Cerebral venous sinus thrombosis (CVT) is a rare cerebrovascular condition accounting for 0.5$1 \%$ of all types of strokes in the general population. Hyperthyroidism is associated with procoagulant and antifibrinolytic activity, thereby precipitating a hypercoagulable state that predisposes to CVT. We report the case of a 31-year-old Korean man with massive CVT and diagnosis of concomitant Graves' disease at admission. Early diagnosis and prompt treatment of CVT are important to improve prognosis; therefore, CVT should be considered in the differential diagnosis in all patients with hyperthyroidism presenting with neurological symptoms.

Keywords: Graves' disease; Hyperthyroidism; Intracranial thrombosis; Thyrotoxicosis

\section{Introduction}

Cerebral venous sinus thrombosis (CVT) is often categorized as a distinct subgroup of cerebrovascular disease, showing an estimated incidence of $0.5-1 \%$ of all kinds of strokes in the general population [1]. CVT is associated with various conditions that result in a hypercoagulable state. Hyperthyroidism is a predisposing factor in approximately $1.7 \%$ of patients with CVT [2]. Notably, 23 papers (including 11 case reports) have discussed the association between thyrotoxicosis and CVT; however, few studies have investigated the pathophysiology of this condition or established a definitive association because of methodological limitations. We describe a patient who presented with massive CVT associated with venous infarction in the right frontal lobe and diagnosis of concomitant Graves' disease.

\section{Case}

A 31-year-old Korean man visited the Emergency Department at Dongsan Hospital with fainting spells and convulsive movements.
He reported a several-day history of recurrent headaches with vomiting, as well as a several-month history of weight loss and heat intolerance. He also reported a history of appendectomy in 1994, short-term use of medications for suspected major depressive disorder in 2012, and surgery for shoulder fracture in 2014.

Initial laboratory investigations revealed normal blood counts, as well as kidney and liver function. Initial brain computed tomography (CT) revealed no definitive evidence of intracranial hemorrhage or detectable low-density infarct-like lesions. Electrocardiography revealed sinus tachycardia. The patient's symptoms worsened the day following his initial visit. Thyroid function tests revealed a serum thyroid-stimulating hormone (TSH) level $<0.01 \mu \mathrm{IU} / \mathrm{mL}$, free thyroxine $4.73 \mathrm{ng} / \mathrm{dL}$, and tri-iodothyronine $378.45 \mathrm{ng} / \mathrm{dL}$. Laboratory tests performed for the assessment of a hypercoagulable state revealed the following results: anti-thyroid peroxidase (anti-TPO) $18.34 \mathrm{IU} / \mathrm{mL}$, thyroglobulin antibody $\mathrm{Ab}$ ) within normal limits, TSH-receptor Ab 14.14 IU/L, d-dimer $5.74 \mu \mathrm{g} / \mathrm{mL}$, fibrinogen $554.5 \mathrm{mg} / \mathrm{dL}$, and factor VIII $210.6 \%$.

Brain magnetic resonance imaging (MRI) was performed based on the emergency protocol followed at our hospital, includ-

Copyright@ 2019 Yeungnam University College of Medicine

This is an Open Access article distributed under the terms of the Creative Commons Attribution Non-Commercial License (http://creativecommons.org/licenses/by-nc/4.0/) which permits unrestricted non-commercial use, distribution, and reproduction in any medium, provided the original work is properly cited. 
ing a sagittal T1-weighted image (T1WI), diffusion-weighted image (DWI), T2 fluid-attenuated inversion recovery (FLAIR) and $\mathrm{T}^{*}$ gradient recalled echo (GRE) sequences. It revealed focal hemorrhagic infarction in the right frontal lobe with venous thrombosis in the superior sagittal (Figs. 1A, 1B) and the right transverse (Fig. 1C) and sigmoid sinuses (not shown in the figure). Indeed, the initial brain CT revealed a subtle hyperdensity lesion on non-enhanced CT images and filling defects in the affected venous sinuses on contrast-enhanced images, particularly partial CVT of the superior sagittal sinus with a contrast-outlined triangular filling defect (empty delta sign) (Figs. 1D-1F). However, these findings were missed during the initial evaluation of im- ages. The patient developed left-sided weakness 3 days after his initial visit. Additional brain CT and MR venography revealed an increased thrombus burden, presenting as significant filling defects in the superior sagittal and the right transverse and sigmoid sinuses (Fig. 2). Previous focal hemorrhagic infarction remained stable without progression, and no additional infarct core or hemorrhagic focus was identified. He received anticoagulation therapy with low-molecular-weight heparin (clexane $60 \mathrm{mg}$ twice a day) on the same day. His left-sided weakness disappeared 3 days after treatment initiation, and headache and nausea also improved 2 days thereafter.

Work-up including thyroid ultrasonography, thyroid scan with
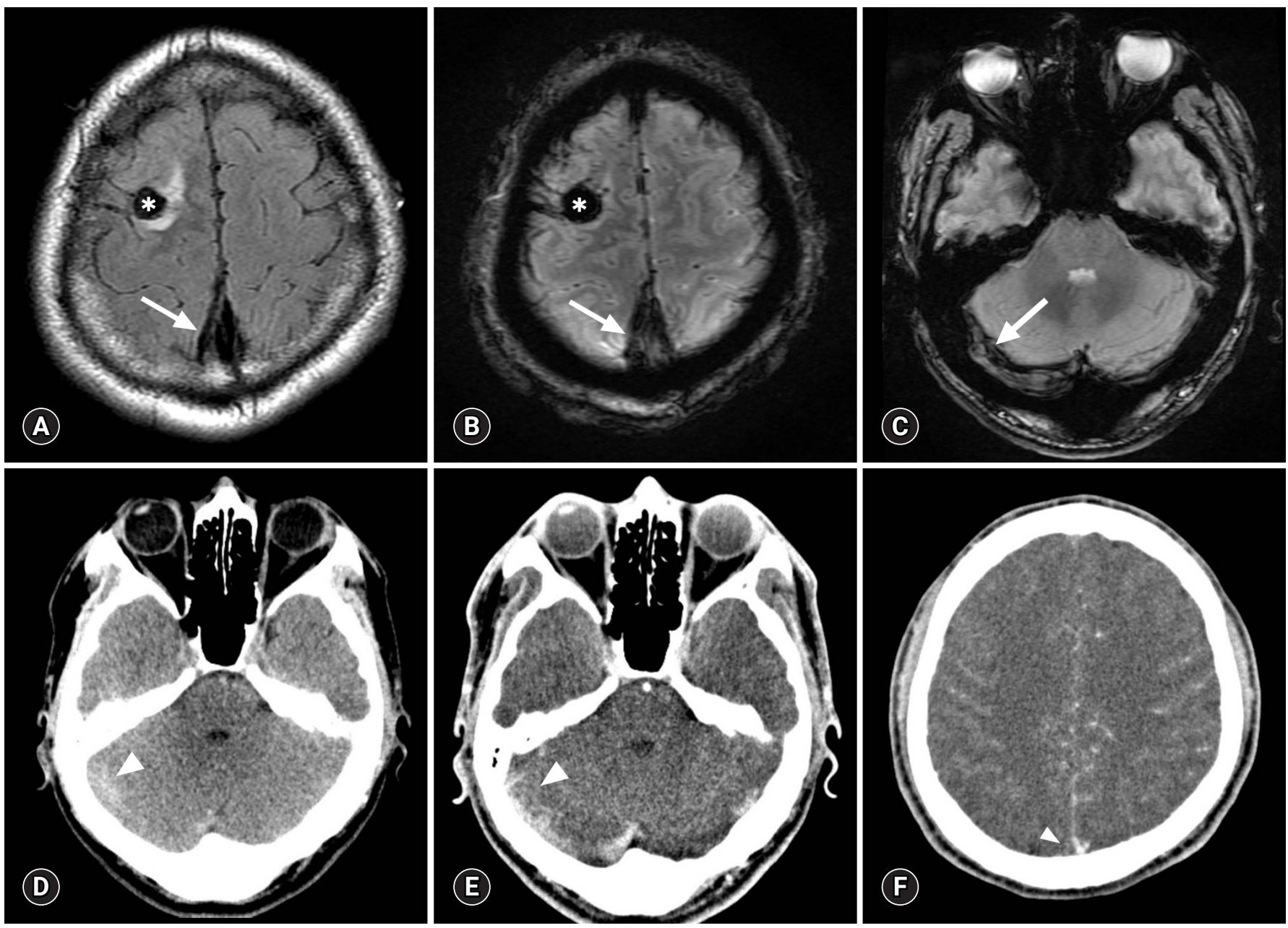

Fig. 1. Initial contrast-enhanced brain CT and MR scans. Focal hemorrhagic infarction is present in the right frontal lobe (asterisk) and a dark signal intensity representing a thrombus (blooming artifact) is present in the superior sagittal sinus (arrows) on the MR scan obtained the following day ( $A$, FLAIR; $B, T 2^{*}$ GRE sequences). A similar dark signal intensity representing a thrombus (arrow) is also present in the right transverse sinus ( $\mathrm{C}, \mathrm{T} 2^{*} \mathrm{GRE}$ ). These findings were neglected on the previous CT scan. The right transverse sinus shows a subtle hyperdensity (arrowhead) on a non-enhanced axial CT scan (D). Contrast-enhanced axial CT scans ( $E_{1}$ F) show corresponding filling defect (arrowhead) at the same location and a partial empty delta sign (arrowhead) in the superior sagittal sinus, which were missed during evaluation of the initial brain CT scan. CT, computed tomography; MR, magnetic resonance; FLAIR, fluid-attenuated inversion recovery; GRE, gradient recalled echo. 
technetium-99m, and laboratory tests confirmed a diagnosis of Graves' disease, and methimazole (antithyroid agent categorized as a TPO inhibitor) was administered at a dose of $12.5 \mathrm{mg}$ twice a day along with indenol (beta blocker) at a dose of $10 \mathrm{mg}$ three times a day. Normalization of thyroid function tests was observed following this treatment.
Follow-up contrast-enhanced CT and MR venography were performed with additional sequences (DWI, T2-FLAIR, T2* GRE) to evaluate residual venous thromboses, 27 days after the patient's initial visit. These imaging studies revealed a significantly reduced thrombus burden in the cerebral deep venous sinuses and partially restored flow enhancement in the right transverse
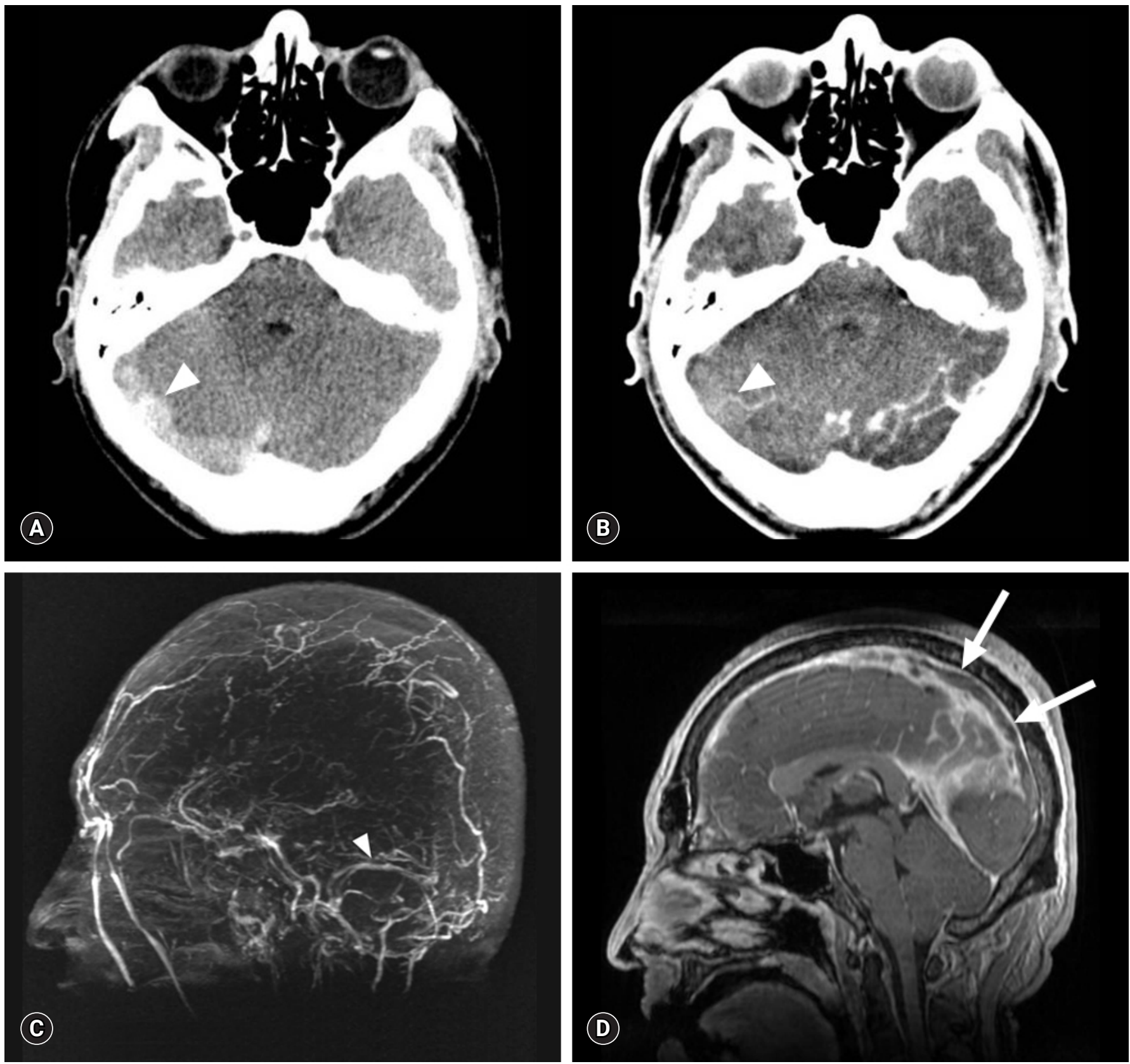

Fig. 2. Brain CT and MR venography scans obtained on admission (3 days after the initial brain CT). Axial non-enhanced CT scan (A) shows a more prominent hyperdensity (HU 70) in the right transverse sinus (arrowhead), which shows the same density (HU 70) on contrast-enhanced brain CT scan (B). Absence of flow-related enhancement is present in the superior sagittal and right transverse to sigmoid sinuses, as opposed to normal flow-related enhancement observed in the left transverse sinus (arrowhead) on an MIP image (C). Sagittal reconstructed MR venography scan (D) shows the propagation of the filling defect along the superior sagittal sinus (arrows). CT, computed tomography; MR, magnetic resonance; HU, Hounsfield units; MIP, maximum intensity projection. 
and sigmoid sinuses without complete recanalization (Fig. 3). At the time of his outpatient follow-up the following month, physical and laboratory examination did not reveal any abnormal neurological findings, and he maintained a euthyroid state. Convention- al MR and CT angiography performed at his 1-year follow-up confirmed near-complete recanalization of the superior sagittal and the right transverse and right sigmoid sinus (Fig. 4).
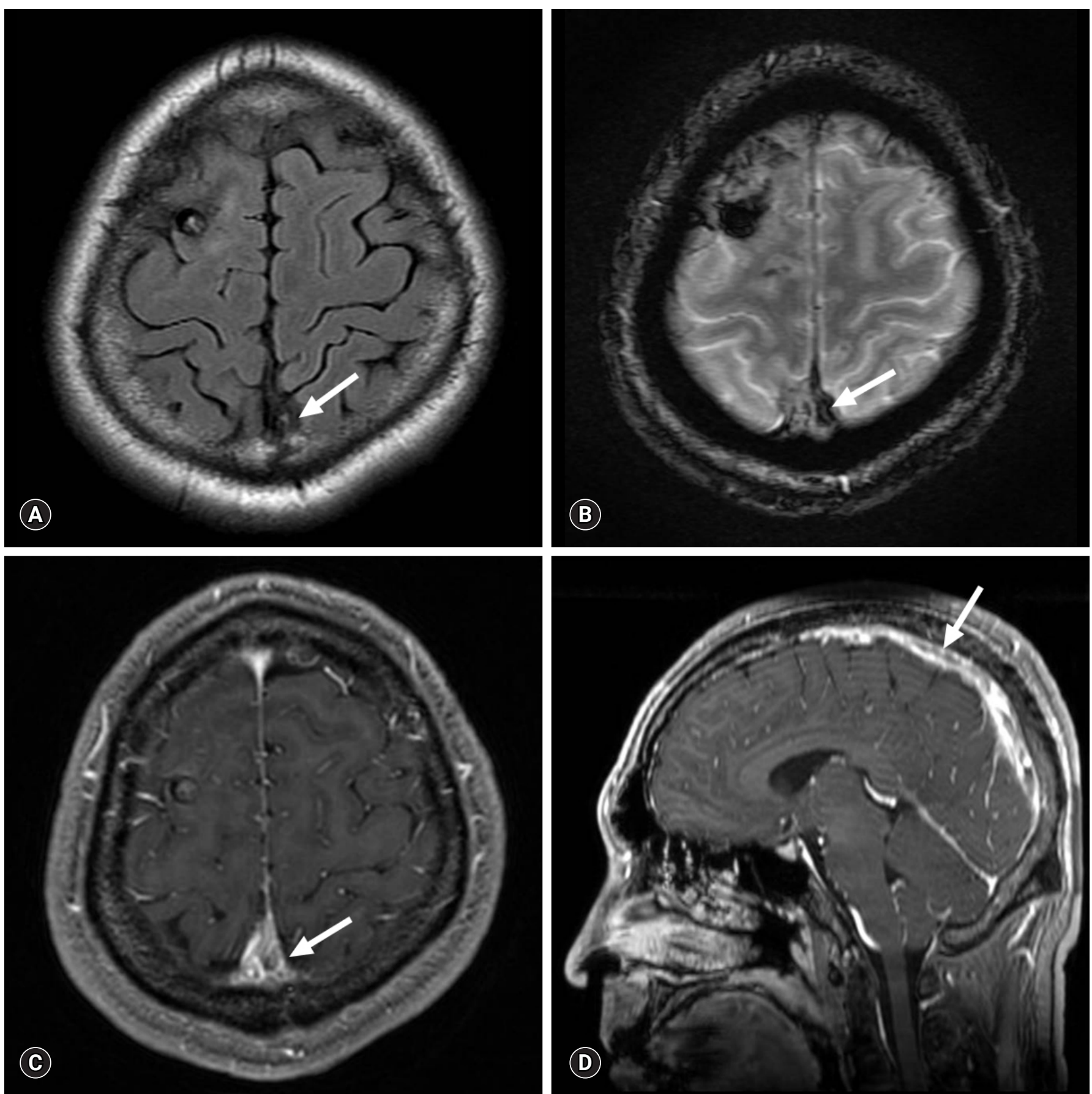

Fig. 3. Conventional and sagittal reformatted MR venography scans obtained at 1-month follow-up. Reduced thrombus burden in the superior sagittal sinus (arrows) is present on axial FLAIR sequence (A), T2* GRE sequence (B), contrast-enhanced T1WI (C), and sagittal reformatted MR venography scan (D). MR, magnetic resonance; FLAIR, fluid-attenuated inversion recovery; GRE, gradient recalled echo; T1WI, T1-weighted image. 


\section{Discussion}

CVT is categorized as a distinct subgroup of cerebrovascular disease, showing an incidence of approximately $0.5-1 \%$ of all kinds of strokes in the general population [1]. CVT is associated with various conditions that result in a hypercoagulable state. Hyperthyroidism is implicated as a predisposing factor for CVT because hyperthyroidism is known to cause elevated procoagulant levels
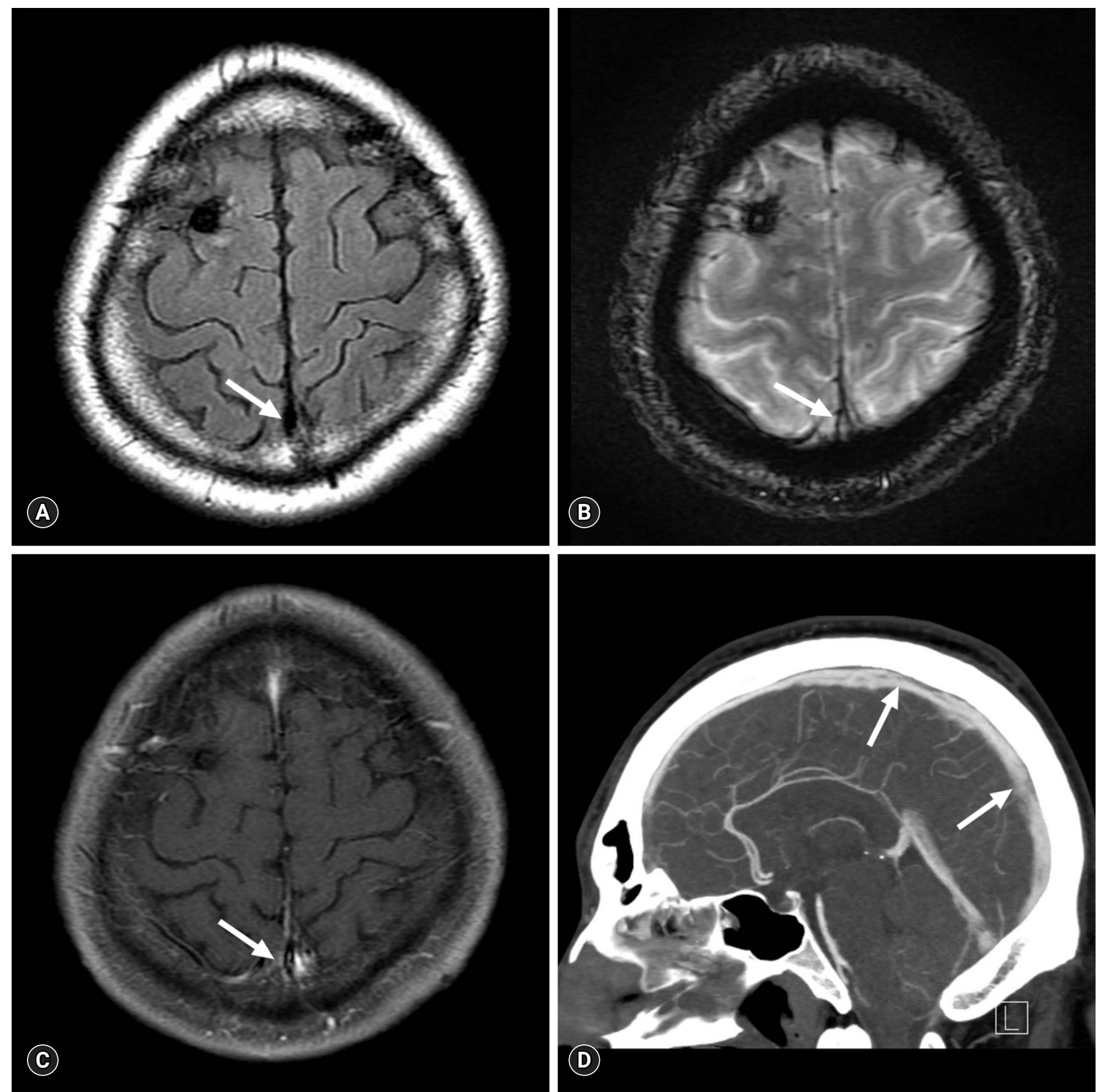

Fig. 4. Conventional $M R$ and delayed-phase $C T$ angiography scans obtained at the last follow-up showing near-complete recanalization of the cerebral venous sinuses. Conventional MR venography scan (A, FLAIR; B, T2* GRE; and C, contrast-enhanced T1WI) and delayedphase CT angiography scan (D) obtained at 1-year follow-up shows near-complete recanalization of the superior sagittal and the right transverse and sigmoid sinuses (arrows). MR, magnetic resonance; CT, computed tomography; FLAIR, fluid-attenuated inversion recovery; GRE, gradient recalled echo; T1WI, T1-weighted image. 
in the vascular hemostatic system leading to a hypercoagulable state. However, to date only several small studies have directly investigated the association between hyperthyroidism and CVT. Diagnostic radiological findings in patients with CVT include the following: venous sinus thrombosis can be identified as a hyperdensity lesion of the affected sinus for the first 7-14 days on non-enhanced CT images. Following contrast administration, CVT presents as a filling defect in a sinus, and patients with involvement of the sagittal sinus typically show the 'empty delta sign.' Reportedly, CT venography shows a sensitivity of $95 \%$ in these cases. MRI can help in visualization of the clot as well as the sequelae. The clot shows variable signal intensity based on its stage (acute-to-chronic stage). During the acute stage, the clot appears isointense on T1WI and hypointense on T2WI, and during the subacute stage the clot appears as a hyperintense lesion on T1WI. Notably, clots can be easily detected as a dark signal within areas of hemorrhage (typically described as blooming artifacts) on T2* GRE or susceptibility images. Venous infarction can be identified on DWI. Contrast-enhanced MR or MR venography shows greater sensitivity in detecting thrombosis, which presents as a venous filling defect in patients with CVT.

The patient described in this case report presented with massive CVT without a medical history to support this diagnosis. Diagnosis of Graves' disease concomitant with CVT was established only at the time of his current admission. The association between CVT and thyrotoxicosis was first described by Kaliebe in 1913 [3] and by Doyle in 1927 [4]; 23 cases have been reported thereafter [5-8]. A hypercoagulable state induced by high circulating levels of thyroid hormones is the likely mechanism contributing to the pathophysiology in these cases [9]. High levels of thyroid hormones increase blood levels of coagulation factors and inhibit fibrinolysis. Notably, increased levels of fibrinogen, von Willebrand factor, coagulation factors VIII, IX and X, and plasminogen activator inhibitor-1 shorten the activated partial thromboplastin time and prolong the clot lysis time [10]. The combined effects of hyperthyroidism on procoagulant and fibrinolytic activity increase the risk of venous thrombosis. Bensalah et al. reported that increased Factor VIII levels were observed in 10 of 26 patients with CVT and concomitant thyrotoxicosis [9]. In our case, the factor VIII level was $210.6 \%$. Currently, venous thrombosis is accepted as a 'multicausal' disease because several genetic and environmental conditions work together [11].

Although initial laboratory investigations revealed poorly controlled thyroid hormone levels indicating thyrotoxicosis, the diagnosis of CVT was delayed in this patient until confirmatory MR venography was performed, because we initially missed the partial empty delta sign in the affected cerebral venous sinuses. Any fur- ther delay in diagnosis could have led to irreversible neurological injury even with optimal treatment. This case emphasizes the importance of careful evaluation for early diagnosis of venous thrombosis in young adults with hyperthyroidism presenting with unexplained neurological symptoms. Clinicians should be aware that further evaluation to confirm CVT should be considered with $\mathrm{CT}$ or MR angiography with venous phase and blood-sensitive MR sequences, such as T2* GRE or susceptibility weighted imaging in patients with a suspicious hyperdensity or the empty delta sign in the cerebral venous sinuses on brain CT.

In conclusion, previously published data strongly suggest that hyperthyroidism should be considered an important risk factor for CVT. Comprehensive evaluation is warranted for prompt diagnosis of CVT in patients with hyperthyroidism presenting with unexplained neurological symptoms.

\section{Conflicts of interest}

No potential conflicts of interest relevant to this article was reported.

\section{ORCID}

Hye-Min Son, https://orcid.org/0000-0003-1748-0701

\section{References}

1. Einhäupl K, Bousser MG, de Bruijn SF, Ferro JM, Martinelli I, Masuhr F, et al. EFNS guideline on the treatment of cerebral venous and sinus thrombosis. Eur J Neurol 2006;13:553-9.

2. Saposnik G, Barinagarrementeria F, Brown RD Jr, Bushnell CD, Cucchiara B, Cushman M, et al. Diagnosis and management of cerebral venous thrombosis: a statement for healthcare professionals from the American Heart Association/American Stroke Association. Stroke 2011;42:1158-92.

3. Kaliebe $\mathrm{H}$. Autochthone sinusthrombose bei einem falle von morbus basedow und tabes. Med Klin 1913;9:1929-32.

4. Doyle JB. Obstruction of the longitudinal sinus. Arch Neurol Psychiatry 1927;18:374-82.

5. Hermans E, Mariën P, De Deyn PP. Sinus sigmoideus thrombosis secondary to graves' disease: a case description. Case Rep Neurol 2011;3:203-9.

6. Hwang JU, Kwon KY, Hur JW, Lee JW, Lee HK. The role of hyperthyroidism as the predisposing factor for superior sagittal sinus thrombosis. J Cerebrovasc Endovasc Neurosurg 2012;14: 251-4.

7. Merino M, Guijarro MG, Iglesias P, Aladro Y, Montero P. Thyro- 
toxicosis and cerebral venous sinus thrombosis, causality or chance alone? Endocrinol Nutr 2012;59:462-3.

8. Migeot M, Rutgers MP, Gille M. Puerperal cerebral sinus venous thrombosis and acute hyperthyroidism in Graves' disease. Acta Neurol Belg 2013;113:331-3.

9. Bensalah M, Squizzato A, Ould Kablia S, Menia H, Kemali Z. Cerebral vein and sinus thrombosis and hyperthyrodism: a case re- port and a systematic review of the literature. Thromb Res 2011;128:98-100.

10. Squizzato A, Romualdi E, Büller HR, Gerdes VE. Thyroid dysfunction and effects on coagulation and fibrinolysis: a systematic review. J Clin Endocrinol Metab 2007;92:2415-20.

11. Rosendaal FR. Venous thrombosis: a multicausal disease. Lancet 1999;353:1167-73. 
